Meta

Journal des traducteurs

Translators' Journal

\title{
Boisson alcoolique ou boisson alcoolisée?
}

\section{Richard Weil-Brenner}

Volume 28, numéro 2, juin 1983

URI : https://id.erudit.org/iderudit/002139ar

DOI : https://doi.org/10.7202/002139ar

Aller au sommaire du numéro

Éditeur(s)

Les Presses de l'Université de Montréal

ISSN

0026-0452 (imprimé)

1492-1421 (numérique)

Découvrir la revue

Citer cet article

Weil-Brenner, R. (1983). Boisson alcoolique ou boisson alcoolisée? Meta, 28(2),

178-186. https://doi.org/10.7202/002139ar d'utilisation que vous pouvez consulter en ligne.

https://apropos.erudit.org/fr/usagers/politique-dutilisation/ 


\section{BOISSON ALCOOLIQUE OU BOISSON ALCOOLISÉE?}

Pour rendre l'expression alcoholic beverage, qu'on emploie en anglais pour désigner «a potable preparation containing ethyl alcohol ${ }^{1}$, c'est-à-dire une boisson qui contient de l'alcool éthylique, ou éthanol ${ }^{2}$, le traducteur dispose de BOISSON ALCOOLIQUE et de BOISSON ALCOOLISÉE, formules en apparence interchangeables, puisqu'aussi bien elles répondent toutes deux à la même définition: "qui contient de l'alcool».

Comment choisir l'une plutôt que l'autre, et dans quel cas, telle est la question à laquelle nous tenterons de répondre.

Notons d'emblée que le problème est complexe et périlleux, et que le risque de se fourvoyer entièrement est considérable. On a répété ad nauseam que les vertus premières du français sont la clarté et la précision. Et La Bruyère, qui aimait le mot juste, nous le rappelle fort à propos: «Entre toutes les différentes expressions qui peuvent rendre une seule de nos pensées, il n'y en a qu'une qui soit la bonne ${ }^{3}$. C'est précisément de cela qu'il s'agit ici : choisir entre deux expressions proposées par le lexique français celle qui traduira le plus justement l'idée exprimée par le syntagme alcoholic beverage.

Selon l'Encyclopaedia Britannica, "Alcoholic beverages include wines, beers and spirits ${ }^{4}$, donc le vin, la bière et les... «spirits?» Dans la New Age Encyclopedia, on définit les «distilled spirits» de la façon suivante : «Alcoholic beverages resulting from the distillation of alcohol-containing liquids. They include brandy, cordials, gin, vodka, rum and whiskey ${ }^{5}$ ". Le Webster's New Twentieth Century Dictionary of the English Language, donne à «spirits" la définition qui suit: «A strong alcoholic liquor produced by distillation ${ }^{6}$ ". Pour «liquor», il donne: "An alcoholic drink, especially one made by distilling, as whisky or rum, as distinguished from such beverages as wine or beer, that have undergone fermentation ${ }^{7} »$. Quant au vin et à la bière, ce sont respectivement «the fermented juice of grapes, used as an alcoholic beverage», et «a mildly alcoholic drink made from any farinaceous grain, but generally from barley, which is first malted and ground, and its fermentable substance then extracted by hot water ${ }^{8}$. Ajoutons, pour que notre liste soit complète, le «cider», défini comme «a sweet fermented liquor" ${ }^{9}$, et le «grog», c'est-à-dire «a mixture of rum and water not sweetened; hence, any kind of alcoholic drink ${ }^{10} »$.

1. McGraw-Hill Dictionary of Scientific and Technical Terms (1978): Second Edition, Montréal. McGraw-Hill Book Company.

2. Encyclopaedia Britannica (1981) : William Benton, Publisher, Chicago : "...the main ingredient that characterizes alcoholic beverages (...) is ethyl alcohol..."

3. La Bruyère, Les caractères, cité par A.L. SARDOU, Nouveau dictionnaire des synonymes français, Librairie Ch. Delagrave, Paris (vers 1925).

4. Op. cit.

5. New Age Encyclopedia (1980) : Lexicon Publications Inc., U.S.A.

6. Webster's New Twentieth Century Dictionary of the English Language (1979), William Collins Publishers, Inc., U.S.A.

7. Ibid.

8. Ibid.

9. Ibid.

10. Ibid. 
Toutes ces boissons «contiennent de l'alcool», soit par suite d'une fermentation (vin, bière, cidre), soit par suite d'une distillation (spiritueux), soit encore par suite d'une addition (grog), et toutes entrent dans la catégorie dite des «alcoholic beverages».

Voyons maintenant ce qu'il en est dans la langue d'arrivée. Nous avons deux expressions consignées dans à peu près tous les dictionnaires : boisson alcoolique et boisson alcoolisée.

Dans ce monument de l'esprit qu'est le Dictionnaire d'Émile Littré ${ }^{11}$, l'adjectif alcoolique signifie «qui contient de l'alcool». Comme exemple d'emploi, Littré propose: «Liqueurs alcooliques, (c'est-à-dire) le vin, l'eau-de-vie et toutes les liqueurs de table. »Quant à alcoolisé, c'est un "participe passé», et il «se dit d'un liquide qui contient de l'alcool ou dans lequel il s'en est développé." le verbe alcooliser a deux sens, dont seul le premier nous intéresse ici ${ }^{12}$ : «Mêler de l'alcool avec un autre liquide." Sous alcoolisme, outre un exemple d'emploi de l'adjectif, Littré nous livre une observation amusante sur les mœurs des ouvriers des pays froids :

Alcoolisme, s.m. Terme de médecine. Alcoolisme chronique, maladie caractérisée par une détérioration graduelle de la constitution et par des accidents nerveux; elle s'observe surtout dans les pays froids, où les travaux pénibles exigent l'emploi des boissons alcooliques de la part des ouvriers; ce qui en conduit beaucoup à abuser de ces boissons.

Comme on le voit, Littré ne distingue pas entre boisson alcoolique et boisson alcoolisée. Toutes les deux «contiennent de l'alcool» et, si l'on en juge par l'exemple d'emploi de l'adjectif et par la définition du participe, on peut conclure qu'il s'agit dans les deux cas d'une boisson dans laquelle il s'est développé de l'alcool: le vin est une liqueur alcoolique, soit une boisson «qui contient de l'alcool» (par fermentation), mais il serait aussi une boisson alcoolisée, car c'est un liquide dans lequel il s'est développé de l'alcool (toujours par fermentation, processus au cours duquel «les enzymes créés par les cellules de levure convertissent le sucre du jus de raisin en alcool... ${ }^{13}$ ».

Nous serions donc en présence d'un cas de synonymie concurrente. Les deux expressions pourraient s'employer l'une pour l'autre selon l'inspiration du moment ou le caprice de chacun. Mais nous allons voir que cette synonymie est tout à fait artificielle et, au demeurant, cousue d'une ambiguïté qui s'est perpétuée pendant un siècle, témoin les exemples qui suivent.

L'Encyclopaedia Universalis ${ }^{14}$ consacre un long article à la maladie de l'alcoolisme. Or les auteurs de cet article emploient tour à tour boisson alcoolique et boisson alcoolisée dans le même sens :

La toxicité des boissons alcoolisées parait bien être due essentiellement à l'éthanol. ...l'absorption régulière $[\ldots]$ de doses relativement importantes de boissons alcooliques (généralement fermentées : vin, bière).

11. LITTRÉ, Émile (1881): Dictionnaire de la langue française, Paris, Librairie Hachette et Cie.

12. Au cas où cela intéresserait quelqu'un, ce second sens est: «Dans l'ancienne pharmacie, réduire en poudre fine, à cause de la signification primitive d'alcool, qui est poudre fine.»

13. LICHINE, Alexis (1980): Encyclopédie des vins et des alcools de tous les pays, Robert Laffont, Paris.

14. Encyclopaedia Universalis (1968) : Encyclopaedia Universalis France, S.A. 
La Grande Encyclopédie Larousse ${ }^{15}$ emboite le pas par l'emploi alternatif de l'une et l'autre expressions :

Alcoolisme: Ensemble de troubles morbides provoqués par une consommation excessive de boissons alcoolisées.

Toutes les boissons alcoolisées (vin, bière, cidre, liqueurs, etc.) peuvent être en cause.

Vocabulaire de l'alcoolisme - Alcoomanie, besoin de boissons alcooliques s'accompagnant d'une impulsion irrésistible à boire; dipsomanie, tendance qui pousse irrésistiblement le sujet à boire des boissons alcoolisées jusqu'à l'ivresse; (...) teetotalisme, système excluant de l'alimentation toute boisson alcoolique (et l'emploi exclusif du thé)...

Les auteurs du Dictionnaire encyclopédique Quillet ${ }^{16}$ ne sont pas plus limpides : Principes à respecter vis-à-vis de l'alcool. - L'abstinence n'est pas nécessaire, mais la sobriété. L'Académie de médecine recommande: [...] pas de boissons alcoolisées en dehors des repas ou à jeun; jamais de boissons alcoolisées pour les enfants. La femme enceinte doit éviter toute boisson alcoolique forte et user modérément (et aux repas seulement) de bière, vin ou cidre.

Dans un document officiel de l'Administration française ${ }^{17}$, on constate encore cette déconcertante synonymie :

...l'intervention de mesures concrètes, facilement réalisables, telles que la distribution de boissons non alcoolisées aux ouvriers...

...de mettre spécialement à leur disposition et à leur portée au moins une boisson non alcoolique...

Les dictionnaires de synonymes ne rapprochent pas les deux expressions, peutêtre, justement, parce qu'elles ne sont pas du tout synonymes, mais ils les emploient eux aussi d'une façon indistincte. Ainsi, le Dictionnaire des synony$m e s^{18}$ de René Bailly nous propose les définitions suivantes :

Alcoolique est moins péjoratif (qu'ivrogne), et se dit [...] de celui qui, sans forcément s'enivrer, fait abus d'une façon habituelle de boissons alcooliques.

«Dipsomane est un terme médical qui sert à désigner un malade chez qui le besoin de boire avec excès des boissons alcoolisées...

Les Synonymes de Bénac ${ }^{19}$ adoptent la même démarche, mais aboutissent à un résultat différent:

Dipsomane, terme médical, malade poussé par accès, sous l'influence d'une impulsion morbide, à boire avec excès des boissons alcooliques.

Le Nouveau dictionnaire des synonymes ${ }^{20}$ de Genouvrier, Désirat et Hordé définit l'alcoolique comme «celui qui boit régulièrement de manière abusive des boissons alcooliques, sans forcément s'enivrer».

Quant aux dictionnaires de langue, s'ils ne sont pas tous aussi déroutants, nous le verrons plus loin, ils nous plongent souvent en pleine pagaille. C'est

15. La Grande Encyclopédie Larousse (1971): Paris, Librairie Larousse.

16. Dictionnaire encyclopédique Quillet (1977) : Paris, Librairie Aristide Quillet.

17. LAURENT, P., Distribution de boissons dans les entreprises, Comité d'Étude et d'Information sur l'Alcoolisme, édité par la Documentation française, Paris, s.d.

18. BAILLY, René (1947): Dictionnaire des synonymes de la langue française, sous la direction de Michel de Toro, Paris, Librairie Larousse.

19. BÉNAC, Henri (1956) : Dictionnaire des synonymes, Librairie Hachette.

20. GENOUVRIER, Émile, Claude DÉSIRAT et Tristan HORDÉ (1977): Nouveau dictionnaire des synonymes, Paris, Librairie Larousse. 
ainsi que le Petit Robert ${ }^{21}$, qui ne se gêne pas pour taxer l'adjectif alcoolique de "vieilli» sans autre forme de procès, ajoute à la confusion. Le premier sens donné au mot alcoolique est «Qui contient de l'alcool». Comme exemple d'emploi, les auteurs proposent : «Les boissons alcooliques ». Le verbe alcooliser a trois sens: $1^{\circ}$ Convertir en alcool. $2^{\circ}$ Additionner d'alcool. (Exemple) : Alcooliser un vin [...] Boisson alcoolisée, qui contient de l'alcool. V. Alcoolique. $3^{\circ}$ Pronom. (Fam.). Abuser des boissons alcooliques, s'enivrer."

Dans le même Petit Robert, sous Alcoolisme, on trouve la définition qui suit: «Abus des boissons alcooliques». Le mot vin est défini comme une «boisson alcoolisée provenant de la fermentation du raisin... » En revanche, la bière est une «boisson alcoolique fermentée», tandis qu'une boisson est «tout liquide qui se boit [...] Boissons alcoolisées [...] Spécialt. Boisson alcoolique...»

Qui disait que l'adjectif alcoolique est vieilli? Notons au passage qu'on ne trouve pas cette marque d'usage dans l'édition de 1981 du Grand Robert ${ }^{22}$, bien qu'elle figure dans le Petit depuis 1975, année même où elle a fait son apparition dans le Trésor de la langue française ${ }^{23}$. S'agit-il d'un oubli? Bien malin qui saura le dire. Quoi qu'il en soit, on observe dans le Grand Robert la même alternance d'emploi des deux expressions : une boisson qui contient de l'alcool est une boisson alcoolique, l'alcoolisme se caractérise par l'abus des boissons alcooliques, une liqueur peut être très alcoolisée... Le verbe alcooliser veut dire mêler de l'alcool à un autre liquide [...] Alcooliser du vin, de la bière...

On pourrait multiplier les exemples presque à l'infini. La documentation pertinente est plus qu'abondante. Les curieux y puiseraient matière à consternation: boisson alcoolique et boisson alcoolisée se côtoient dans la plus joyeuse des synonymies. Et pourtant... n'a-t-on pas déjà dit pourtant qu'il n'existe pas de synonymes parfaits; qu'il y a des nuances de sens, des colorations de contexte, des subtilités d'atmosphère, des parfums, des odeurs et des textures qui rendent impossible l'interchangeabilité aveugle des mots et expressions d'une même langue? Et que, dès lors, on ferait bien d'exercer son jugement dans l'emploi qu'il est permis d'en faire?

Comme tous les synonymes français, (ces termes) ne sont pas parfaitement interchangeables. Il faut donc user de discernement et choisir l'expression juste selon le contexte ${ }^{24}$.

Ce à quoi on peut ajouter: selon la bonne intelligence des choses à nommer. Tous les bonnets blancs ne sont pas forcément blancs bonnets.

Les auteurs du Trésor de la langue française ont-ils jugé que la confusion avait assez duré? Peut-être; en tout cas, ils se sont prononcés: l'adjectif alcoolique, dans leur édition de 1975, est marqué du signe du «vieillissement», avec la précision suivante : «En parlant d'un liquide». Et pour montrer claire-

21. ROBERT, Paul (1975-1981): Dictionnaire alphabétique et analogique de la langue française, Paris, Société du nouveau Littré (Petit Robert).

22. ROBERT, Paul (1981): Dictionnaire alphabétique et analogique de la langue française, Paris, Société du nouveau Littré. (Grand Robert).

23. IMBS, Paul (1975) : Trésor de la langue française, Tome quatrième, Paris, Éditions du Centre national de la recherche scientifique.

24. SANSREGRET, Louise (1979): les Mots dits Grands Maux, Montréal, Société d'énergie de la Baie James. 
ment qu'ils n'ont pas pris leur décision à la légère, ils citent des exemples d'emploi de deux auteurs du XIX $\mathrm{X}^{\mathrm{e}}$ siècle, sans doute pour faire encore plus vieux : Jules Michelet (1798-1874) et Jules Verne (1828-1905). Puis ils ajoutent: «Alcoolisé concurrence fortement alcoolique. On dit plutôt boisson alcoolisée que boisson alcoolique. "

Fort bien, mais la deuxième acception consignée dans le Trésor pour l'adjectif alcoolique se lit comme suit: "Qui est provoqué par les boissons alcooliques: Moréas en ce moment souffre de crises alcooliques et n'est pas à côté de son ancien collaborateur. »Et plus loin, encore ceci : «B.-Adj. ou subst. (En parlant d'une pers.) (Celui) qui est intoxiqué par les boissons alcooliques.»

Qui a dit que «le vin délie la langue?» On croirait plus volontiers qu'à son commerce elle fourche! Car enfin, si la question était de démontrer que l'adjectif alcoolique (en parlant d'un liquide) est «vieilli», il ne devrait pas suffire de citer des auteurs d'un autre siècle. Et il ne faudrait pas l'employer sans vergogne soi-même; et il faudrait en outre que le mot fût disparu de l'usage depuis longtemps, ce qui est loin d'être le cas. Comme les auteurs du Trésor ne justifient d'aucune façon cette marque d'usage (pas plus, au reste, que les auteurs du Petit Robert), mais ne font que la consigner très arbitrairement, on est en droit de se demander s'ils ne succombent pas tout simplement devant une certaine mode qui répugne à employer un mot auquel il serait attaché, à ce qu'il paraît, une connotation péjorative. Cela se peut, mais ce n'est pas très sérieux. Surtout si l'on se rappelle que Grévisse souligne le fait que le suffixe ique est «le plus employé pour former des adjectifs et notamment des adjectifs de la terminologie scientifique $25 \%$. Signalons à cet égard que nous avons relevé quatre-vingt-trois adjectifs formés au moyen du suffixe -ique dans les soixantehuit pages (couvertures comprises) du numéro 882 du 3 octobre 1981 du Nouvel Observateur. Chacun de ces quatre-vingt-trois adjectifs y est employé au moins une fois.

Nous avons aussi relevé moult exemples d'emploi modernes de l'expression «boisson alcoolique». Nous reproduisons ci-dessous une liste partielle des documents dans lesquels nous l'avons rencontrée. Précisons que dans aucun de ces documents elle n'était accompagnée de sa «jeune» concurrente.

Canada, Statistique Canada, Annuaire du Canada, 1975, p. 773;

Bibliothèque de l'Université Laval, Section de l'analyse documentaire, Division du Traitement, Répertoire de vedettes-matières, 8e édition, Québec, 1976, p. 99;

Chartrand, Luc, «Les souverains déchus» Protégez-vous, publiée par l'Office de protection du consommateur, Éditions Protégez-vous Inc., Saint-Laurent, août 1981, p. 5;

Craplet, Camille, et Josette Craplet-Meunier, Dictionnaire des Aliments et de la nutrition, Le Hameau, Paris, 1979, p. 55;

France, Code des débits de boissons et des mesures contre l'alcoolisme, édition mise à jour au 30 mai 1978, Première Partie (Législative), Dispositions applicables aux boissons, Article L. 1, p. 1;

Hôtellerie Restauration, Vol. 55, n ${ }^{\circ} 3$, Montréal, mars 1981, p. 4;

La Presse, Cahier spécial «Vins et liqueurs fines», Montréal, 1974, p. 3;

Mantoux, Paul, la Révolution industrielle au XVIII siècle, Éditions Genin, Paris, 1959, p. 443 ;

25. GRÉVISSE Maurice (1980) : le Bon Usage, 11" édition, Paris-Gembloux, Éditions Duculot. 
Ministère des Affaires culturelles, Bibliothèque nationale du Québec, Radar, Vol. 8, $\mathrm{n}^{\circ}$ 6, 1980, p. A-121;

Québec, Gazette officielle du Québec, Partie II, Index des textes réglementaires au 31 décembre 1980, Éditeur officiel du Québec, p. 113.

Mentionnons que les fabricants québécois de spiritueux, de vin et de cidre se sont réunis en septembre 1980 en "Association de l'industrie manufacturière de boissons alcooliques du Québec».

Pour intéressantes qu'elles soient, ces considérations sur la valeur d'emploi de l'adjectif alcoolique (en parlant d'un liquide) ne permettent pas d'élucider entièrement le problème. Notons que les auteurs du Petit Robert avaient d'ailleurs mis le lecteur en garde, quant à l'interprétation qu'il convient de faire des marques d'usage :

Il va de soi que cette notation généralisée des valeurs d'emploi est délicate et sujette à contestations: certains pourront trouver courants des termes que nous aurons considérés comme techniques ou scientifiques, d'autres emploieront (ou auront l'impression d'employer) des mots notés comme vieillis ${ }^{26}$.

Il faut donc encore sonder la réalité que nous cherchons à nommer. D'après les définitions données par Adolphe $\mathrm{V}$. Thomas 27 , «Un liquide alcoolique contient naturellement de l'alcool», (tandis que) "Alcoolisé se dit d'un liquide auquel on a ajouté de l'alcool: Boire une tisane alcoolisée.» On trouve quelque chose de singulièrement approchant dans le Pluridictionnaire Larousse 28 : «Alcoolique, adj. Qui contient de l'alcool», et «Alcoolisé, e-adj. Auquel on a ajouté de l'alcool: Une bière fortement alcoolisée."

Louis-Alexandre Bélisle ${ }^{29}$ propose des définitions analogues : «Alcoolique, adi. qui contient de l'alcool», et «Alcooliser, v.a. Mêler de l'alcool avec un autre liquide.»Ces acceptions de l'adjectif (ou du participe) alcoolisé et du verbe alcooliser rejoignent celle consignée dans The Heritage Dictionary of the English Language $^{30}$ : «Alcoholize - tr. v. - ized, izing, izes. To saturate, mix or treat with alcohol». Le Grand Larousse de la langue française ${ }^{31}$ donne des définitions qui sont de la même veine : «Alcoolique, adj. Qui est à base d'alcool. Une liqueur alcoolique. Se dit d'une personne qui fait abus des breuvages alcooliques», (mais) «Alcoolisé, e-se dit de ce qui est mêlé d'alcool [...] boisson alcoolisée»; et "Alcooliser» (veut dire) ajouter de l'alcool à un autre liquide ou à une préparation: vous n'avez pas assez alcoolisé ce grog...»

Dans le dictionnaire $\operatorname{Logos}^{32}$, le terme alcoolisation signifie $1^{\circ}$ Transformation en alcool: alcoolisation du sucre par la fermentation alcoolique. $-2^{\circ}$ Addition d'alcool à une substance. - $3^{\mathrm{e}}$ Etat de l'organisme d'une personne qui abuse

25. GRÉVISSEd Maurice (1980) : le Bon Usage, 11e édition, Paris-Gembloux, Éditions Duculot.

26. REY, Alain, op. cit., Présentation du dictionnaire.

27. THOMAS, Adolphe V. (1971): Dictionnaire des difficultés de la langue française, Paris, Librairie Larousse.

28. Pluridictionnaire Larousse (1975) : Paris, Librairie Larousse.

29. BÉLISLE, Louis-Alexandre (1971): Dictionnaire général de la langue française au Canada, $2^{e}$ éd., Québec, Bélisle Éditeur.

30. MORRIS, William (1973): The Heritage Dictionary of the English Language, Boston, American Heritage Publishing Co., Inc. et Houghton Mifflin Company.

31. GUILBERT, L., R. LAGAVE et N. GEORGES (1971): Grand Larousse de la langue française, Paris, Librairie Larousse.

32. GIRODET, Jean (1976): Logos, Grand dictionnaire de la langue française, Rennes, Bordas. 
habituellement de l'alcool, sans pour autant être vraiment alcoolique (on dit aussi «imprégnation alcoolique»). Le mot alcoolisé s'entend pour un produit «qui contient de l'alcool; auquel on a ajouté de l'alcool: boisson alcoolisée; médicament faiblement alcoolisé. " Enfin, alcooliser veut dire " $l^{\circ}$ Transformer en alcool: la fermentation alcoolise le jus de raisin. $-2^{\circ}$ Ajouter de l'alcool à une substance: alcooliser un médicament», et s'alcooliser signifie «s'intoxiquer par l'abus des boissons alcooliques." Le même dictionnaire définit alcoolique comme un adjectif ou un nom. En tant qu'adjectif, il signifie $I^{\circ}$ Relatif à l'alcool, spécialement à l'alcool éthylique. - $2^{\circ}$ Qui contient de l'alcool éthylique: boisson alcoolique (fermentée, comme le cidre, le vin, ou distillée, comme l'eau-de-vie). $-3^{\circ}$ Qui est atteint d'alcoolisme ». Employé substantivement, il désigne «Un, une alcoolique.»

De toutes ces définitions, il ressort que l'adjectif alcoolique, employé «en parlant d'un liquide», n'a qu'un sens: qui contient de l'alcool, et qu'il s'applique à toutes les boissons qui se sont alcoolisées par suite d'une fermentation ou d'une distillation (bière, vin, cidre, spiritueux, eaux-de-vie, liqueurs), tandis que l'adjectif alcoolisé s'entend principalement pour un liquide auquel on a ajouté de l'alcool et accessoirement, par extension du sens du participe, pour un liquide ou une boisson qui contient de l'alcool. Dans son premier sens, il s'applique à toute boisson additionnée d'alcool (café avec cognac ou whiskey, jus d'orange avec vodka ou tout autre mélange d'une boisson non alcoolique avec une boisson alcoolique); dans son second sens, il s'emploie dans un sens purement technique, pour nommer le résultat du processus d'alcoolisation. C'est donc une acception qu'il convient de réserver aux ouvrages spécialisés.

Pour ce qui est des boissons alcooliques proprement dites, voyons comment le docteur Camille Craplet, médecin nutritionniste et maitre de conférence à l'I.N.A., en opère la classification.

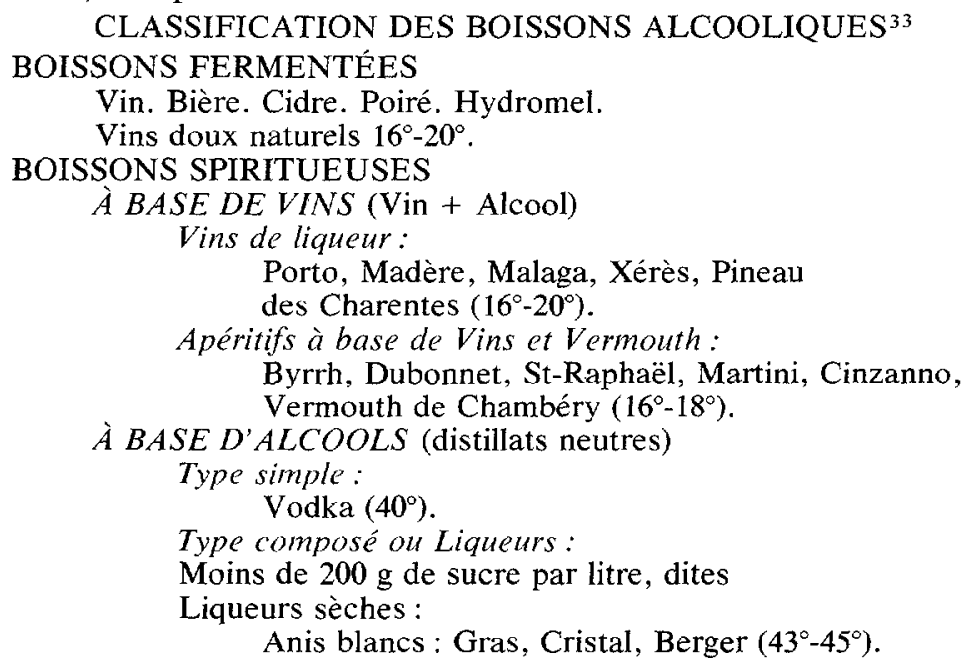

33. CRAPLET, Camille, et Josette CRAPLET-MEUNIER (1979) : Dictionnaire des aliments et de la nutrition, Paris, Le Hameau. 


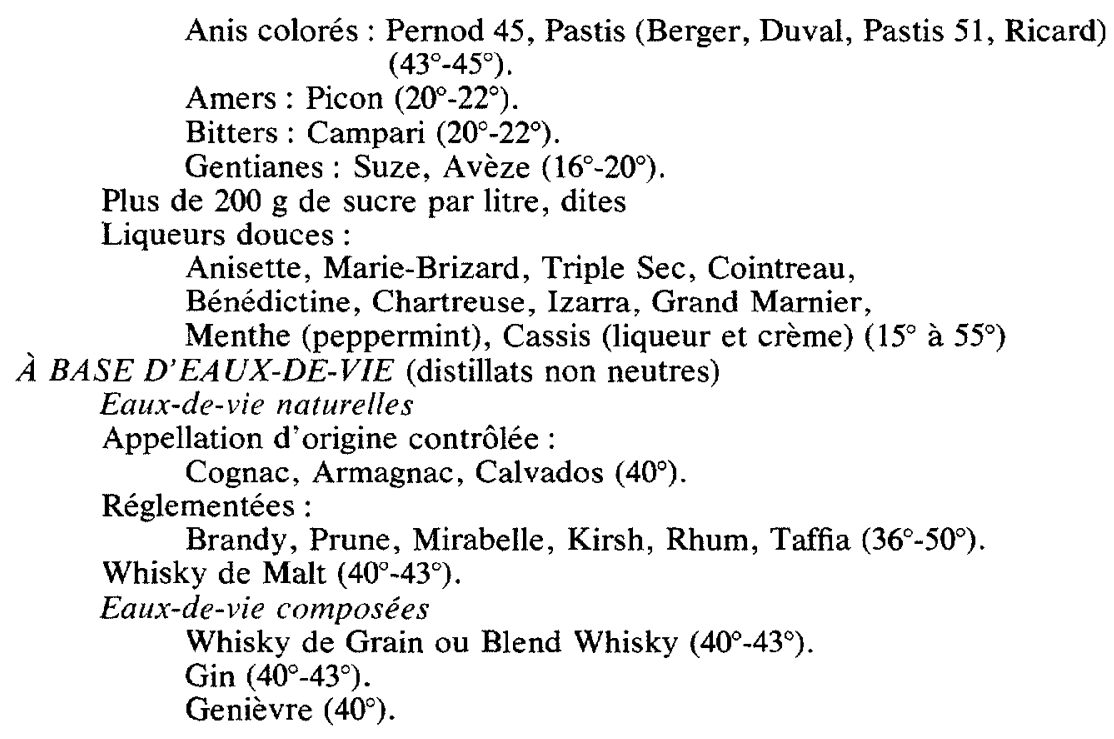

Pour ce qui est des boissons alcoolisées (boissons additionnées d'alcool, le Dictionnaire universel de cuisine pratique ${ }^{34}$ et le Nouveau Larousse gastronomique 35 en proposent de nombreuses recettes, dont le clairet, l'hypocras à la framboise, le grog, de même que toute une série de boissons réunies sous le nom de punch.

La distinction entre boisson alcoolique et boisson alcoolisée est donc évidente. Citons, pour conclure, quelques auteurs qui se sont penchés sur la question d'une façon spécifique : Dupré, Colin, Girodet, Laurin et Thomas.

É. DUPRÉ - «Comme adjectif (alcoolique), il s'applique aux liquides comme le vin, le cidre, la bière, les liqueurs qui proviennent de fruits ou de céréales ayant subi une fermentation. Un liquide alcoolisé est celui auquel on a ajouté de l'alcool: un grog, par exemple, où l'eau-de-vie est ajoutée à de l'eau chaude sucrée et à du citron. On peut aussi alcooliser un vin en lui ajoutant de l'alcool ${ }^{36}{ }_{»}$.

J.P. COLIN • "ALCOOLIQUE ou ALCOOLISÉ - Emploi et sens. Une boisson alcoolique contient de l'alcool, par fermentation naturelle. Une boisson alcoolisée est un liquide additionné d'alcool. Le vin est une boisson alcoolique; un grog est une boisson alcoolisée ${ }^{37} »$.

J. GIRODET • «Alcoolique, alcoolisé - Deux adjectifs à bien distinguer.

1. Une boisson alcoolique, qui contient de l'alcool naturellement, par ellemême (vin, bière, cidre, eau-de-vie, apéritif, liqueur, etc.).

2. Une boisson alcoolisée, que l'on confectionne en versant de l'alcool dans un liquide (cas du grog, par exemple) ${ }^{38} »$.

34. FAVRE, Joseph (1978): Dictionnaire universel de cuisine pratique, tome 1, Marseille, Laffite Reprints.

35. MONTAGNÉ, Prosper (1967): Nouveau Larousse gastronomique, édition revue et corrigée par Robert J. Courtine, Paris, Librairic Larousse.

36. DUPRÉ, Émile (1972): Encyclopédie du bon français dans l'usage contemporain, Paris, Éditions de Trévisse.

37. COLIN, Jean-Paul (1970): Nouveau dictionnaire des difficultés du français, collection dirigée par Henri Mitterand, Paris, Éditions Hachette-Tchou.

38. GIRODET, Jean (1981): Dictionnaire du bon français, Paris, Bordas. 


\section{J. LAURIN • Alcoolique et alcoolisé}

Alcoolique signifie qui contient de l'alcool. Les boissons alcooliques: gin, vodka, scotch, etc.

Alcoolisé signifie additionné d'alcool. Un café alcoolisé (dans lequel on a ajouté du cognac, par exemple) ${ }^{39} 》$.

Adolphe V. THOMAS $\bullet$ Alcoolique - alcoolisé. - Un liquide alcoolique contient naturellement de l'alcool. Il ne faut pas abuser des boissons alcooliques.

"Alcoolisé se dit d'un liquide auquel on a ajouté de l'alcool: Boire une tisane alcoolisée $e_{\Downarrow \text {. }}^{40}$.

Si l'on adopte le point de vue exprimé par ces auteurs, il convient de réserver l'emploi de l'expression boisson alcoolisée aux seules boissons auxquelles on a ajouté de l'alcool. En effet, dans la mesure où l'on ne retient que cette acception restreinte de l'adjectif alcoolisé, il n'y a pas lieu, sous peine de commettre une bévue, de dire que le cognac est une boisson «alcoolisée», car c'est une boisson «alcoolique».

Au sens large, néanmoins, le cognac est bel et bien une boisson alcoolisée, car c'est un liquide dans lequel il s'est développé de l'alcool, d'abord par fermentation, puis par distillation. Mais on remarque tout de suite que le cognac s'est alcoolisé non pas par addition d'alcool, mais parce qu'il a subi le processus d'alcoolisation. Et comme il s'est transformé en alcool, c'est encore et toujours une boisson alcoolique. On l'appellera boisson alcoolisée si l'on veut, mais dans un cadre technique ou scientifique seulement.

Dans le domaine de la restauration, par contre, il est bien évident que la distinction entre boisson alcoolique et boisson alcoolisée est capitale. Non seulement rend-elle justice aux exigences de clarté et de précision de la langue française, mais elle ressortit à la bonne intelligence de la réalité. Quand la liste des boissons offertes à la clientèle comporte des vins, des bières et des spiritueux, on se gardera de l'intituler "Liste des boissons alcoolisées". C'est, dans ce contexte, «Liste des boissons alcooliques» qu'il faut dire. Si, d'aventure, un client réclame une boisson alcoolisée, il est de bon ton de lui en demander respectueusement la recette.

RICHARD WEIL-BRENNER 